\title{
A climate network perspective on the intertropical convergence zone
}

\author{
Frederik Wolf ${ }^{1,2}$, Aiko Voigt ${ }^{3,4}$, and Reik V. Donner ${ }^{1,5}$ \\ ${ }^{1}$ Research Domain IV - Complexity Science, Potsdam Institute for Climate Impact Research (PIK) - \\ Member of the Leibniz Association, Potsdam, Germany \\ ${ }^{2}$ Department of Physics, Humboldt University, Berlin, Germany \\ ${ }^{3}$ Institute of Meteorology and Climate Research, Department Troposphere Research, Karlsruhe Institute \\ of Technology, Karlsruhe, Germany \\ ${ }^{4}$ Lamont-Doherty Earth Observatory, Columbia University in the City of New York, NY, USA \\ ${ }^{5}$ Department of Water, Environment, Construction and Safety, Magdeburg-Stendal University of Applied \\ Sciences, Magdeburg, Germany
}

Correspondence: Frederik Wolf (frederik.wolf@pik-potsdam.de)

Received: 21 October 2020 - Discussion started: 6 November 2020

Revised: 21 February 2021 - Accepted: 23 February 2021 - Published: 31 March 2021

\begin{abstract}
The intertropical convergence zone (ITCZ) is an important component of the tropical rain belt. Climate models continue to struggle to adequately represent the ITCZ and differ substantially in its simulated response to climate change. Here we employ complex network approaches, which extract spatiotemporal variability patterns from climate data, to better understand differences in the dynamics of the ITCZ in state-of-theart global circulation models (GCMs). For this purpose, we study simulations with 14 GCMs in an idealized slab-ocean aquaplanet setup from TRACMIP - the Tropical Rain belts with an Annual cycle and a Continent Model Intercomparison Project. We construct network representations based on the spatial correlation patterns of monthly surface temperature anomalies and study the zonal-mean patterns of different topological and spatial network characteristics. Specifically, we cluster the GCMs by means of the distributions of their zonal network measures utilizing hierarchical clustering. We find that in the control simulation, the distributions of the zonal network measures are able to pick up model differences in the tropical sea surface temperature (SST) contrast, the ITCZ position, and the strength of the Southern Hemisphere Hadley cell. Although we do not find evidence for consistent modifications in the network structure tracing the response of the ITCZ to global warming in the considered model ensemble, our analysis demonstrates that coherent variations of the global SST field are linked to ITCZ dynamics. This suggests that climate networks can provide a new perspective on ITCZ dynamics and model differences therein.
\end{abstract}

\section{Introduction}

One-third of Earth's precipitation falls within the narrow band of the deep tropics within $10^{\circ} \mathrm{N}-\mathrm{S}$ (Kang et al., 2018). This narrow band is home to the intertropical convergence zone (ITCZ), in which the northerly and southerly trade winds of the Hadley circulation meet and give rise to surface convergence of moist air, ascent, and hence rainfall (Wallace and Hobbs, 2006). Because tropical rainfall is critical to many societies and ecosystems, reliable projections of the ITCZ response to climate change are key to mitigation and adaptation efforts in a warming world (Donohoe and Voigt, 2017). Yet, global climate models are affected by persistent biases in the simulation of tropical rainfall and the ITCZ in the present-day climate, have difficulties in capturing past ITCZ shifts, and show limited consensus on how the ITCZ location, width, and strength will change in response to increasing atmospheric carbon dioxide levels (Bony et al., 2015; Harrison et al., 2015; Byrne et al., 2018). These model difficulties reflect the importance of small-scale cloud pro- 
cesses and their coupling with the large-scale circulation, e.g., via cloud radiative effects (Voigt et al., 2014) and convective mixing (Moebis and Stevens, 2012), and have motivated a large amount of theoretical and idealized work (Kang et al., 2009; Donohoe et al., 2013; Schneider et al., 2014; Biasutti et al., 2018). This has led to important insights into how the position of the ITCZ is controlled by atmospheric energy transport and sea surface temperatures (SSTs).

Generally speaking, heating one hemisphere relative to the other leads to an ITCZ shift into the heated hemisphere because of the cross-equatorial atmospheric energy transport required to balance the hemispheric heating (Kang et al., 2009; Donohoe et al., 2013). Moreover, warming the surface of one hemisphere also leads to an ITCZ shift into the warmer hemisphere, in line with changes in nearsurface moist static energy and boundary-layer convergence (Lindzen and Nigam, 1987; Emanuel et al., 1994). These considerations have formed our understanding of how the ITCZ is connected to the spatial pattern of atmospheric energetics and SST. These perspectives have been employed to study the movement of the ITCZ on seasonal timescales (e.g., Adam et al., 2016) as well as on the longer timescales of past and future climate change (e.g., Donohoe et al., 2013). They have further been used to understand the response of the ITCZ to specific perturbations from, for example, aerosols, ocean heat transport, and clouds (e.g., Hwang et al., 2013; Frierson et al., 2013; Voigt et al., 2014, 2017) and for investigating model shortcomings in simulating the ITCZ (Adam et al., 2018). Still, in many cases the success of these perspectives is limited, and their link to the ITCZ in model simulations can be weaker than expected (Biasutti and Voigt, 2020). In the context of the present study it is also important to remember that they operate in a time average sense: they link the time average ITCZ position to the time average atmospheric energy transport and time average SST pattern. The time average can mean both a seasonal mean and a long-term annual mean.

In this study, we aim to test an alternative perspective based not on the time average SST field but on its variability. We apply tools from complex network theory and account for the information encoded in the spatiotemporal variability of the SST pattern, which we attempt to relate to the time average ITCZ position. Different from the frameworks described above, our network approach links spatial correlation patterns of the global SST field to the time mean ITCZ position. For this purpose, we employ the concept of functional climate network analysis (Tsonis and Roebber, 2004; Donner et al., 2017; Dijkstra et al., 2019) that focuses on the strongest mutual statistical interdependencies among spatially distributed records of climate variability. While being based on the same correlation matrix as popular linear analysis approaches in statistical climatology such as empirical orthogonal function (EOF) analysis, this approach involves a nonlinear filter that highlights only key structures and makes their associated spatial interdependence pat- terns fully transparent (Donges et al., 2015b; Donner et al., 2017). As a result, functional climate networks have found a rising variety of applications in climate science (Dijkstra et al., 2019). Among others, they have provided key insights into the climate dynamics associated with large-scale tropical SST anomalies representing the oceanic manifestation of the El Niño-Southern Oscillation. Specifically, the finding that climate network patterns based on global surface air temperature anomalies are significantly affected by El Niño and La Niña episodes (Yamasaki et al., 2008; Gozolchiani et al., 2008, 2011) has led to the development of improved strategies for El Niño forecasting (Ludescher et al., 2013, 2014; Feng et al., 2016; Meng et al., 2020) and a selfconsistent classification of different flavors of El Niño and La Niña based on their corresponding global imprints (Radebach et al., 2013; Wiedermann et al., 2016). Other successful applications include the development of early warning indicators for a possible collapse of the Atlantic Meridional Overturning Circulation based on ocean temperature correlations (van der Mheen et al., 2013), uncovering key spatiotemporal patterns associated with heavy precipitation formation in different monsoon regions (Malik et al., 2012; Boers et al., 2013, 2014; Stolbova et al., 2014), improved forecasting of the Indian summer monsoon onset and rainfall amount (Stolbova et al., 2016; Fan et al., 2020), and identification of teleconnection pathways (Zhou et al., 2015; Boers et al., 2019). In all these examples, spatiotemporal climate data have been transformed into a network representation based on different concepts of statistical association used for identifying mutually dependent climate time series.

To better understand the effect of spatiotemporal SST patterns on ITCZ dynamics, we present the first application of functional climate network analysis to idealized aquaplanet simulations from the TRACMIP (Tropical Rain belts with an Annual cycle and a Continent Model Intercomparison Project) model ensemble (Voigt et al., 2016) that is freely available via the Earth System Grid Federation and the Pangeo project. The simulation setup and the corresponding data are briefly introduced in Sect. 2, which also details our analysis methodology that combines functional climate network analysis with a hierarchical clustering method to classify the TRACMIP models according to their climate network topology. We focus on two questions. First, to what extent is the network-based classification related to the models' ITCZ position in the control simulation? And second, to what extent is the response of the ITCZ to quadrupled atmospheric carbon dioxide related to changes in the climate network? The obtained results are presented in Sect. 3. The paper closes with a discussion and conclusion in Sect. 4. 


\section{Data and methods}

\subsection{TRACMIP model ensemble}

TRACMIP provides a suite of simulations with 14 global circulation models in an idealized aquaplanet setup and a setup with an idealized continent. TRACMIP was designed to study fundamental aspects of the ITCZ and its response to climate change, e.g., the link of the ITCZ to SST and crossequatorial atmospheric energy transport (Biasutti and Voigt, 2020). TRACMIP can also be used in a much broader sense, including studies of phenomena such as the Arctic amplification (Russotto and Biasutti, 2020). The TRACMIP protocol has been described in detail in Voigt et al. (2016), which includes references for the participating models.

The most salient feature of TRACMIP compared to other aquaplanet studies is the use of a slab ocean with a presentday-like ocean heat transport and seasonally varying insolation. In TRACMIP, sea surface temperatures are thus interactive and the surface energy balance is closed, the ITCZ migrates north and south during the year, and the ITCZ is located in the Northern Hemisphere in the zonal mean and time average, consistent with the present-day climate.

In this work, we use aquaplanet simulations performed by all 14 models contributing to the intercomparison project. The AquaControl simulation is run with a present-day-like $\mathrm{CO}_{2}$ concentration of $348 \mathrm{ppmv}$. In the Aqua $4 \times \mathrm{xCO} 2$ simulation, $\mathrm{CO}_{2}$ is quadrupled to $1392 \mathrm{ppmv}$, leading to a modeldependent increase in global mean surface temperature by $3-10 \mathrm{~K}$ and changes in the ITCZ location that range from a slight southward shift to strong northward shifts by up to $8^{\circ}$ in latitude. Following Biasutti and Voigt (2020), the ITCZ position is calculated by the precipitation centroid as defined in Adam et al. (2016) over latitudes within $20^{\circ} \mathrm{N}-$ $\mathrm{S}$. This locates the ITCZ somewhat closer to the Equator compared to the values diagnosed in Voigt et al. (2016), but the results of our analysis are insensitive to the definition of the ITCZ position. Figure 1 illustrates the precipitation and sea surface temperature pattern of the AquaControl simulations. This figure also demonstrates the tight correlation of the ITCZ position with the tropical SST contrast (correlation coefficient $>0.99$ ). Again following Biasutti and Voigt (2020), the latter is defined as the SST difference between the Northern and Southern Hemisphere tropical means (Equator-25 $\mathrm{N}$ and $25^{\circ} \mathrm{S}-$ Equator, respectively).

Figure 1 confirms that SST is a prime control on the ITCZ, a fact that has been exploited in many previous studies. However, in contrast to previous work that used the time average SST, we apply functional climate network representations to study the relation between the ITCZ and the internal variability of the SST field. An illustration of the temporal SST variability is shown in Fig. 2 for the AquaControl simulation. The SST variability is minimal near the Equator, is relatively constant in the subtropics and midlatitudes, and drops off near the poles. This pattern is broadly in line with the vari- ability in the sum of surface turbulent fluxes (sensible and latent heat flux) and surface winds (not shown).

The TRACMIP model output is provided on regular latitude-longitude grids that have a model-dependent horizontal resolution ranging from 1 to $3^{\circ}$ in latitude and longitude. For the AquaControl simulations, we restrict our analysis to the last 30 years and for the Aqua4xCO2 simulations to the last 25 years to ensure that the models are in statistical equilibrium. As described below, we focus on monthly mean SST fields, from which we construct functional climate networks and study their relation to the time average ITCZ position and tropical SST contrast.

\subsection{Functional climate networks}

Functional climate networks constitute an application of complex network theory to understand functional relations in the Earth's climate system. In general, complex networks often serve as abstract mathematical models of complex systems, in which individual entities are represented by nodes that are connected by links symbolizing interdependencies among the entities. In climate networks nodes are identified with geographical locations at which climate variability information is available in the form of time series (in our case, the grid points of climate model outputs), and links connect pairs of nodes whose climate time series exhibit a certain level of statistical association. In this work, we analyze the set of SST time series on the longitude-latitude grid separately for each model and simulation run using the methodological setup described in the following.

First, we calculate the linear Pearson correlation coefficient for the monthly SST anomalies of all pairs of grid points. The time series of the SST anomalies are computed by subtracting the monthly mean climatology from the original SST time series. In principle, we could utilize any statistical association measure in this step. We chose the Pearson correlation here as it has been successfully applied in previous studies (Donges et al., 2009; Radebach et al., 2013; Wiedermann et al., 2016) and is suitable for relatively short time series (here, 300 to 360 monthly anomaly values). For a model output with $N$ grid points, this leads to a correlation matrix $\mathbf{S}$ of dimension $N \times N$.

Second, we threshold the correlation matrix and transform it into a binary matrix $\mathbf{A}$. $\mathbf{A}$ is the adjacency matrix of the associated climate network representation of the underlying data set and has the same dimension as $\mathbf{S}$. If the correlation value $s_{i j}$ between two grid point time series (nodes) $i$ and $j$ is larger than the prescribed threshold, we set the corresponding matrix element of $\mathbf{A}$ to $a_{i j}=1$ (i.e., there is a link between nodes $i$ and $j$ ) and to $a_{i j}=0$ otherwise (no link). We choose the correlation threshold in such a way as to obtain a link density of $\rho=0.005$. This implies that the network only includes the strongest $0.5 \%$ of all possible $N(N-1) / 2$ undirected links (when excluding self-correlations, i.e., $a_{i i}=0$ ). For global networks with a comparable resolution, this order 

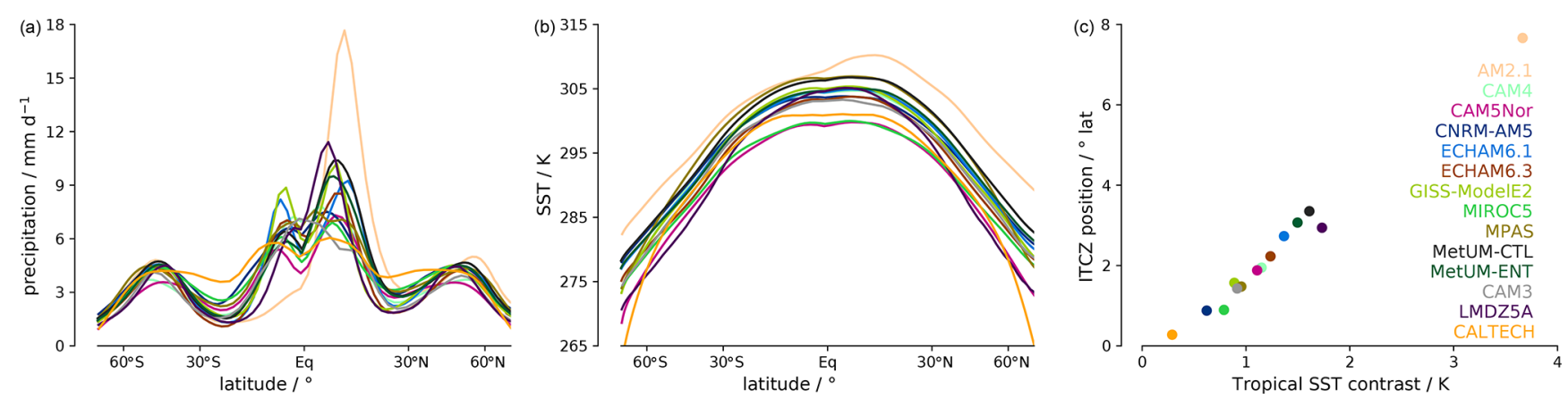

Figure 1. Time average zonal-mean precipitation (a) and surface temperature (b) in the AquaControl simulations. (c) The tight correlation of the time average ITCZ with the SST contrast between the Northern and Southern Hemisphere tropics.

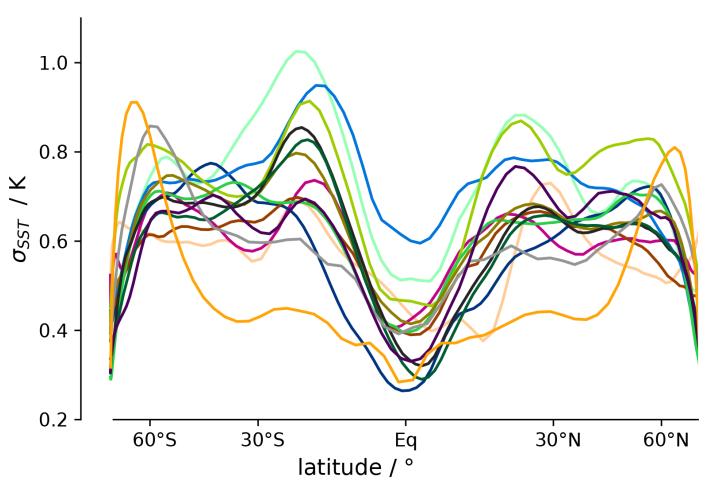

Figure 2. Zonal mean of the temporal standard deviation of monthly mean SST in the AquaControl simulations. The models are color-coded as in Fig. 1.

of magnitude has been a common choice in previous studies (Radebach et al., 2013; Donner et al., 2017). It has to be noted, however, that changes in the link density of a climate network have been previously shown to potentially result in qualitatively different behaviors of the resulting network characteristics (Radebach et al., 2013; Wiedermann et al., 2017b) (depending on the specific network property under study). For this reason, we keep the link density fixed for all analyses performed in this work. However, since the individual models differ in their spatial grid resolution, the total number of links will differ between models, with a lower number of links in the case of coarser grids. This has direct consequences for the quantitative analysis of the resulting networks. In the following, we will choose our analysis setup such that the effect of a different number of links on the final results is minimized.

Third, based on the adjacency matrix we calculate the values of two network characteristics: the degree and the average link distance. The degree $k_{i}$ encodes the number of links of each node $i$,

$k_{i}=\sum_{i=1}^{N} a_{i j}$, where the index $i$ corresponds to a specific latitude-longitude grid point. As our climate networks are spatially embedded on regular spherical grids, the spatial density of nodes on a spherical surface is not homogeneous and increases towards the poles. To make all nodes equally representative, we utilize the concept of the so-called node-splitting invariance (NSI; Heitzig et al., 2012) and define the accordingly nodeweighted NSI degree as

$k_{i}=\sum_{i=1}^{N} w_{i} a_{i j}$.

Here, $w_{i}$ is the NSI node weight, which in our case is given by the cosine of the latitude. For simplicity, in the remainder of this work, we will briefly refer to the NSI degree (sometimes also termed area-weighted connectivity; Tsonis et al., 2006; Tsonis and Swanson, 2008) as the degree. Moreover, instead of discussing the full spatial pattern of degree on the whole sphere, we will focus on the zonal-mean degree. The meaningful calculation and interpretation of this property are ensured by the zonally uniform boundary conditions of the aquaplanet setup. To compare the zonal-mean degree pattern between models despite their differences in grid resolution, we normalize the zonal-mean degree such that its sum over all latitudes is 1 .

The information provided by the purely topological measure of node degree is complemented by the average link distance, which is a spatial (geometric) characteristic. The average link distance is the mean great circle distance between a given node and all its connected nodes (i.e., the mean spatial length of all links of a given node). For simplicity, the average link distance is given here in units of radians; physical distances could be easily obtained by scaling the values with the Earth's radius.

We emphasize that we have also studied a suite of additional local (such as the local clustering coefficient) and global network properties (such as the network transitivity) (Radebach et al., 2013; Donner et al., 2017). These measures have not provided additional insights and are therefore not reported in the following. 


\subsection{Hierarchical clustering}

After having constructed the network for each individual model and simulation, we study to what extent the models can be grouped according to the resulting patterns of their zonal-mean network measures. For this purpose, we employ a hierarchical cluster analysis.

As a first step, we resample the zonal-mean network measure distributions to the lowest latitudinal resolution of all models $\left(\mathrm{CALTECH}, 2.8^{\circ}\right)$ utilizing linear interpolation. Then, we calculate the Pearson correlation coefficient between (selected parts of) the values of the resampled zonalmean network measures as a function of the latitude between all pairs of models. Since the Pearson correlation is invariant under rescaling and possible additive terms, offsets between the individual models' magnitudes of the zonal-mean network measures that originate from the different grid resolutions do not impact the correlation values.

We calculate the Pearson correlation individually for both the degree and the average link distance and then sum the correlation values for each model pair. This results in a matrix $\mathbf{C}$ of dimensions $14 \times 14$ with elements representing the pairwise similarity between all 14 models in terms of both types of zonal-mean network measures. The similarity measure can therefore exhibit values in the range $[-2,2]$ (i.e., the sum of two values each bounded by $[-1,1])$. For convenience, we transform this into normalized values within the interval $[0,1]$ by employing a linear rescaling:

$$
c_{i j}^{\text {new }}=\frac{c_{i j}-\min C}{\max _{i j}\left(c_{i j}-\min C\right)},
$$

with $\mathbf{C}_{\text {new }}$ being the rescaled inter-model similarity matrix that is then used as an input for the hierarchical cluster analysis. Note that $\min C=\min _{i j} c_{i j}$ and $\max C=\max _{i j} c_{i j}$ are the minimal and maximal values among all inter-model correlations and that $\min C$ can hence be negative.

Finally, we cluster models by means of the hierarchical cluster analysis as implemented in the Python package scipy, whereby we use the single linkage method for successively combining groups of models according to the highest pairwise correlation among the included models. This methodological choice ensures that the most similar models are grouped into the same cluster, yet it allows for the possibility to produce outliers. With the number of considered models being quite low, we can at every iteration of the algorithm identify such outliers and explain this by the properties of the employed cluster analysis method while ensuring that the most similar models indeed end up in the same cluster. Specifically, based on the models' mutual similarity, our hierarchical cluster analysis method iteratively identifies pairs of models that are successively merged into clusters until all models belong to a single cluster. The order of this clustering is depicted in a dendrogram. In the visual representation used in this work, the resulting dendrograms are meant to be read from left (each individual model constitutes a single cluster) to right (all models are combined into one cluster). Vertical lines indicate a merge of two models or clusters of models, while the horizontal lines represent the increasing cophenetic distance between the clusters (capturing the degree of similarity between the two groups based on the rescaled intermodel similarity matrix). Cutting the dendrogram at a certain level of similarity, i.e., cophenetic distance, leads to certain clusters of models that will then be used in the remainder of this study.

We are aware that there are alternative ways to quantify the similarity between different models based on the values of the different network measures and to cluster the models. Our methodological choices reflect the need to account for differences in the grid resolution of the 14 global climate models and our preference for a simple and intuitive analysis setup.

\subsection{Robustness tests}

We acknowledge that our analysis setup is based on several specific choices of methodological parameters or variants. To address this, we have tested our results for robustness in the following manner. Due to the limited amount of data and only 14 models to be clustered, we have performed two basic tests on the results. First, we have split the 30 years into two 15-year periods and analyzed the two sets of anomaly time series individually. Although the results are not identical and do not completely match the results from the analysis of 30 years, the clustering of the models is only marginally affected (see Supplement Sect. S1). Second, we have stacked the time series of 20 randomly chosen years and compared the resulting zonal-mean network measures for 20 independent realizations. We have found that the main features of the zonal-mean degree distribution are retained on average among the resulting network ensembles (see Sect. S2). The two sensitivity tests hence indicate that the results described below are robust.

Regarding the cluster analysis employed for grouping the TRACMIP models according to the mutual similarity of their respective network representations, there is a large body of alternative methodological approaches, including hierarchical clustering techniques with different linkage strategies (e.g., complete linkage, average linkage, or Ward's method), partitioning methods like $k$-means clustering, spectral clustering, and many more. To account for the corresponding variety of options and highlight the relevance of an educated choice of the method, we have also considered the complete and average linkage methods (see Sect. S3). The resulting commonalities and differences with respect to single linkage clustering will be discussed for the example of the AquaControl simulations. 


\section{Results}

In the following, we present the results of our functional network analysis. We first make use of the AquaControl simulations and start by investigating climate networks constructed from the complete (global) SST field that include both tropical and extratropical nodes (Sect. 3.1). Subsequently, we study networks for which some of the regional connections were excluded (Sect. 3.2). Finally, we study the response of the climate networks and the ITCZ position to a quadrupling of $\mathrm{CO}_{2}$ by repeating the analysis for the Aqua4xCO2 simulations (Sect. 3.3).

\subsection{AquaControl simulations: global network properties}

The global network analysis is shown in Fig. 3 and separates the models into four clusters (left panel), with clusters 1 and 2 each consisting of six models and cluster 3 and cluster 4 each containing only a single model. The zonal-mean network measures that underlie this clustering are shown in Fig. 4 and discussed in more detail below. Importantly, Fig. 3 demonstrates that the clustering successfully separates models in terms of their time average tropical SST contrasts (central panel) and, to a slightly lesser extent, ITCZ positions (right panel). Specifically, the models in cluster 1 exhibit a stronger SST contrast and a more poleward-shifted ITCZ than the models in cluster 2. Indeed, there is no overlap between the two clusters regarding the respective SST contrast and ITCZ position. The clustering also separates models in terms of the strength of their Southern Hemisphere Hadley circulation as measured by the magnitude of the minimum of the mass stream function (cluster 1: 130-158; cluster 2: 102-129; cluster 3: 53; cluster 4: 266; all values in units of $10^{9} \mathrm{~kg} \mathrm{~s}^{-1}$ ). This is expected since the ITCZ position and the Hadley cell strength tend to be strongly correlated (Donohoe et al., 2013).

The zonal-mean network measures of the four clusters are presented in Fig. 4; the left panels show the normalized degree and the right panels the average link distance. The spatial patterns of the zonal-mean network measures differ systematically between the clusters along with their respective ITCZ positions and SST contrasts. This consistency demonstrates that hierarchical clustering is indeed climatologically meaningful. Despite some inter-model variability in each cluster, clusters 1 and 2 exhibit systematic differences. For cluster 1 (Fig. 4, first row), all models show a coherent degree and link distance minimum around the position of the ITCZ and a marked peak of both measures related to a strong Southern Hemisphere Hadley cell. This finding has already been reported by Wolf et al. (2019) and reflects the fact that the models in cluster 1 have the strongest Southern Hemisphere Hadley cell across the model ensemble. For cluster 2, the zonal-mean average link distance exhibits a broad maximum around the ITCZ position instead of the minimum found for cluster 1 (Fig. 4, second row). More- over, the zonal-mean network measures of cluster 2 are more symmetric with respect to the Equator than for the models in cluster 1 . The comparison between clusters 1 and 2 reveals that a more northward ITCZ position tends to be associated with less symmetric network properties and minima in degree and link distance near the ITCZ, whereas an ITCZ closer to the Equator is accompanied by a more symmetric pattern of zonal-mean network characteristics with only small meridional contrasts in the tropical degree and a nearequatorial maximum in the average link distance.

As opposed to the aforementioned two groups of models, the networks derived from the CALTECH and AM2.1 models, which form cluster 3 and cluster 4, respectively, show zonal-mean network measures that resemble extreme versions of cluster 1 (for AM2.1) and cluster 2 (for CALTECH). CALTECH (cluster 3) has the ITCZ closest to the Equator among all models, and its network characteristics exhibit nearly perfect symmetry with respect to the Equator. By contrast, for AM2.1 (cluster 4) the ITCZ is shifted far into the Northern Hemisphere and its zonal-mean network characteristics exhibit hardly any symmetry with respect to the Equator, yet there are marked minima of degree and average link distance at about the latitudinal position of the ITCZ.

We further demonstrate the success of the functional climate network analysis along with the hierarchical cluster analysis in the following manner. For all pairs of models, we plot the inter-model difference in the ITCZ position and tropical SST contrast as a function of the similarity of the models' zonal-mean network characteristics. The latter is measured by the elements of the original (non-rescaled) intermodel similarity matrix, which represents the combined similarity of the patterns of the two considered zonal-mean network characteristics (see Sect. 2.3). Here, we note again that the matrix elements $c_{i j}$ are bounded to the interval $[-2,2]$ because the values of the Pearson correlation coefficients for zonal-mean degree and zonal-mean average link distance are both restricted to $[-1,1]$. Figure 5 shows the resulting scatter plots of the pairwise similarity coefficient versus the ITCZ position and tropical SST contrast, respectively.

Despite the generally large scatter, there is a clear tendency towards smaller differences in the ITCZ position and SST contrast for models whose climate networks are more similar (Fig. 5). Put differently, this underlines the fact that for models that are identified as more similar by the network and cluster analysis (larger correlation score on the $x$ axis), the pairwise difference in the ITCZ position and SST contrast tends to be smaller than for models with less similar network characteristics. In addition, all combinations of model pairs that belong to cluster 1 or cluster 2 (recall that cluster 3 and cluster 4 only include only one model each) are colored in red and blue, respectively. This shows that indeed the clustering only groups models together that have similar network characteristics.

In the analysis described above, we have utilized a hierarchical cluster analysis with the single linkage approach. 

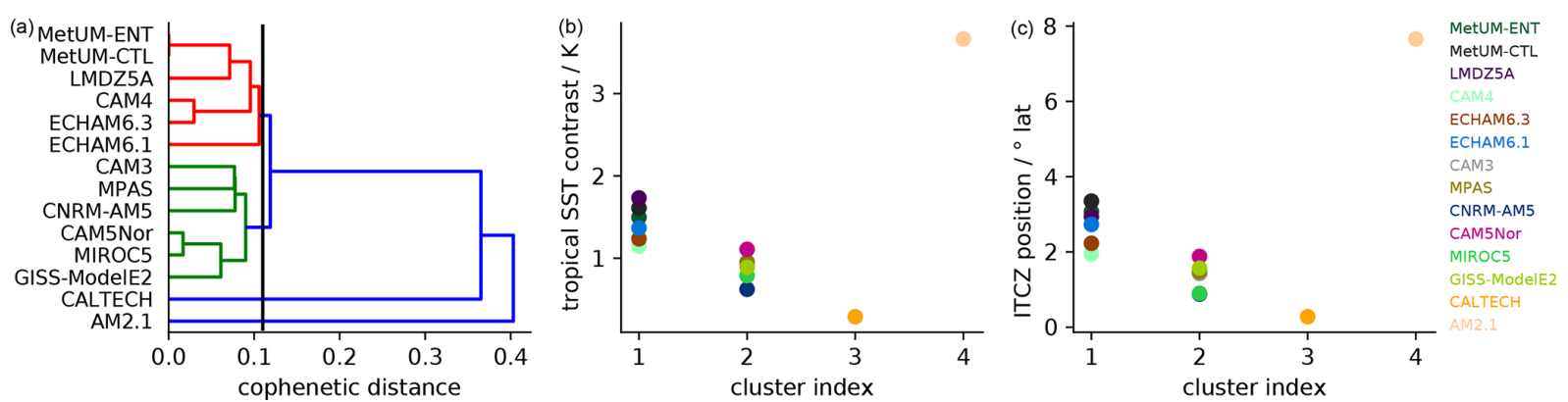

Figure 3. Model clustering of the AquaControl global networks (a) along with the tropical SST contrast (b) and ITCZ position (c) for the four identified clusters. (a) The dendrogram obtained from the clustering of the zonal-mean network measures. The vertical line indicates the level of cophenetic distance at which we split the models into four clusters.
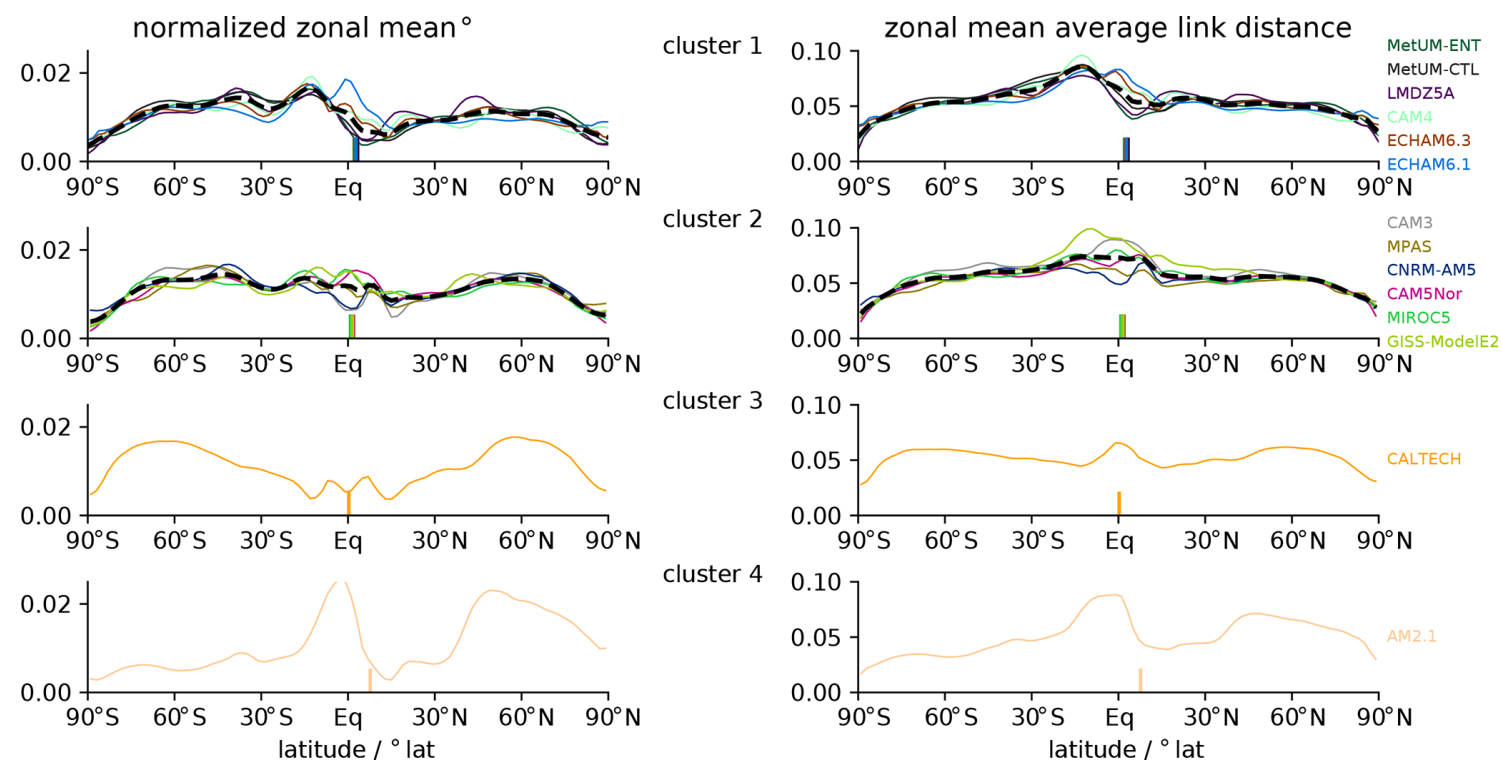

Figure 4. Zonal-mean network measures from the global networks of the AquaControl simulations for each of the four clusters. The left panels show the normalized zonal-mean degree, and the right panels show the corresponding average link distance patterns. Vertical lines indicate the position of the ITCZ. The cluster mean is shown by black dashed lines.

In general, there are a plethora of other methodological options for clustering entities. Among the hierarchical approaches, the existing methods differ in their respective criteria for merging elements or groups of elements. For example, one common alternative to the single linkage approach is the complete linkage method, whereby the largest (in our case cophenetic) distance among pairs of elements is considered. By contrast, the average linkage technique evaluates the mean of all pairwise distances among models belonging to two different groups. An application of both mentioned alternative linkage methods leads to substantial differences in the resulting group structure, although certain pairs of models are categorized into the same subgroup as under the single linkage method (see Sect. S3). This finding could be a direct consequence of the specific distribution of similarity scores (see Fig. 5). The minor differences in mutual similarity among the models in the single-linkage- based clusters illustrate the sensitivity of a clustering procedure considering the mean of all pairwise similarities (single model pairs with reduced similarity might essentially influence the average group similarity and the largest cophenetic distance between group members). As already mentioned, there would be many further alternatives of hierarchical or non-hierarchical clustering methods beyond the two aforementioned ones. In general, we can expect that due to the rather small size of the studied model ensemble, it is of minor interest for the purpose of this study to analyze multiple clustering criteria more systematically (seeking some abstract statistical optimality). We have therefore chosen to focus on the hierarchical single linkage approach in all following analyses, since it allows for a straightforward interpretation of the resulting dendrograms.

In summary, our above results show that functional climate network analysis, although only using information from 


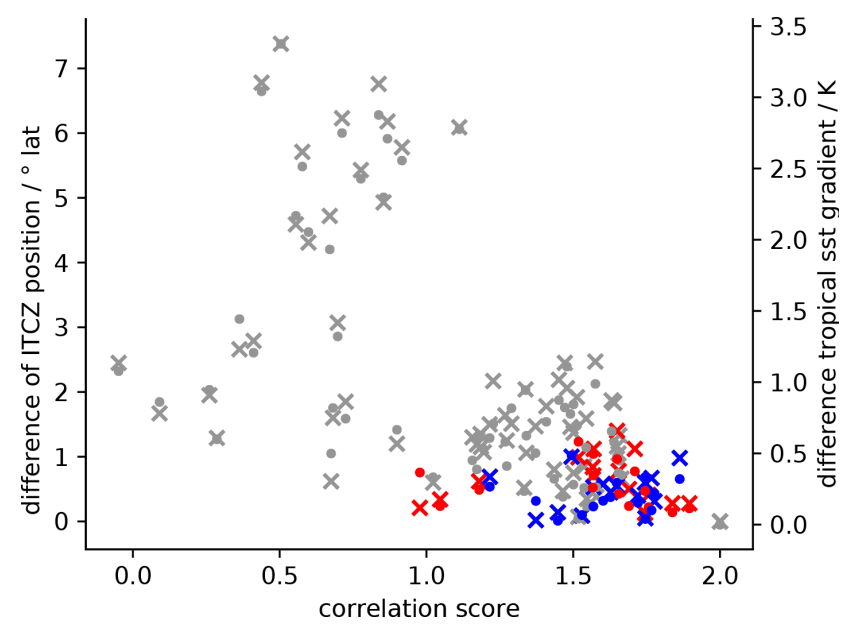

Figure 5. Pairwise inter-model correlation score versus the difference in the ITCZ position and SST contrast of all model pairs. Model pairs with both models falling into the same multi-model cluster are colored in red (cluster 1) and blue (cluster2), respectively. The difference in the ITCZ position is marked by circles; the difference in the SST contrast is marked by " $x$ ".

monthly variability of the global SST field, is able to distinguish time average model differences in the ITCZ position, SST contrast, and Hadley cell strength.

\subsection{AquaControl simulations: networks with stepwise exclusion of regional connections}

The global network analysis in the previous subsection included both tropical and extratropical connections. To disentangle the relative importance of tropical and extratropical connections, we repeat the analysis but successively exclude different classes of links by setting the corresponding elements of the adjacency matrix to zero. Notably, this strategy removes links from the network, whereas the zonal network measures are attributes of nodes, and each link contributes to the degree and average link distance of two different nodes. Hence, we still retain complete zonal-mean characteristics of networks with a specific subset of links. The analysis of the residual network structures thereby allows us to quantify the importance of, e.g., connections within the tropics or between the tropics and the extratropics.

First, we remove all trans-equatorial connections between the Northern and Southern Hemisphere extratropics $\left(>35^{\circ} \mathrm{N}-\mathrm{S}\right)$. This analysis leads to almost indistinguishable results compared to the global networks (not shown) because the number of trans-equatorial extratropical-extratropical connections is very low for all models. This shows that inter-hemispheric teleconnections between the Northern and Southern Hemisphere extratropics do not markedly affect the ITCZ position.

Second, we exclude all extratropical-extratropical connections (i.e., also links connecting two nodes within the ex- tratropics of the same hemisphere). As a consequence, the resulting network characteristics in the extratropics only include tropical-extratropical connections, while the network measures in the tropics feature both tropical-tropical and tropical-extratropical connections. This leads to a very low link density in the extratropics, while the link density in the tropics remains almost as large as in the analysis of the complete network. The extratropics of both hemispheres together account for $110^{\circ}$ in latitude that contribute to the zonal-mean values (with low link density and an irregular distribution), while the tropics cover only $70^{\circ}$ in latitude (but with high link density and a smooth distribution). As a consequence, correlations between the irregular distributions in the extratropics do not lead to meaningful results, while the patterns of zonal-mean network measures in the tropics also include the information from extratropical-tropical links. In the following, we therefore only consider zonal-mean network characteristics between $35^{\circ} \mathrm{S}$ and $35^{\circ} \mathrm{N}$ to quantify the similarity between the respective model outputs. By considering the zonal-mean characteristics only for this tropical band, we obtain smooth and stable patterns that represent both innertropical and tropical-extratropical connections.

The dendrogram obtained by our hierarchical cluster analysis as well as the associated SST contrasts and ITCZ positions are shown in Fig. 6. Unlike for the complete network, the analysis without extratropical-extratropical connections separates the models into four clusters of similar size. Three of the four clusters differ regarding their SST contrast, ITCZ position, and Southern Hemisphere Hadley cell strength. The network analysis thus also identifies some model differences in the tropical climate when extratropical-extratropical connections are removed, but the success in doing so is reduced compared to the analysis of the global network.

The zonal-mean network measures underlying the clustering in Fig. 6 are shown in Fig. 7. The models in cluster 3 (third row) exhibit minima of the zonal-mean network characteristics near the ITCZ and maxima in the region of the Southern Hemisphere Hadley cell. A similar feature is to some extent visible for the models in cluster 1 (first row), although it is blurred by features like additional local minima and marked maxima of both network properties in the region of the Northern Hemisphere Hadley cell. The network measures for models in cluster 2 (second row) exhibit rather heterogeneous distributions, consistent with the relatively large model spread in SST contrast and ITCZ position in that cluster. Finally, the network properties of the models in cluster 4 (bottom row) feature some level of symmetry with respect to the Equator, although their distributions differ substantially and their cophenetic distance in the dendrogram is large (Fig. 6 left). In line with the previous results, we notice that a decrease in the level of symmetry in the network measures is associated with an ITCZ that is more strongly shifted into the Northern Hemisphere. This is expected, as for the ITCZ to be shifted away from the Equator, there needs to be some level of hemispheric asymmetry in the SST pattern. 

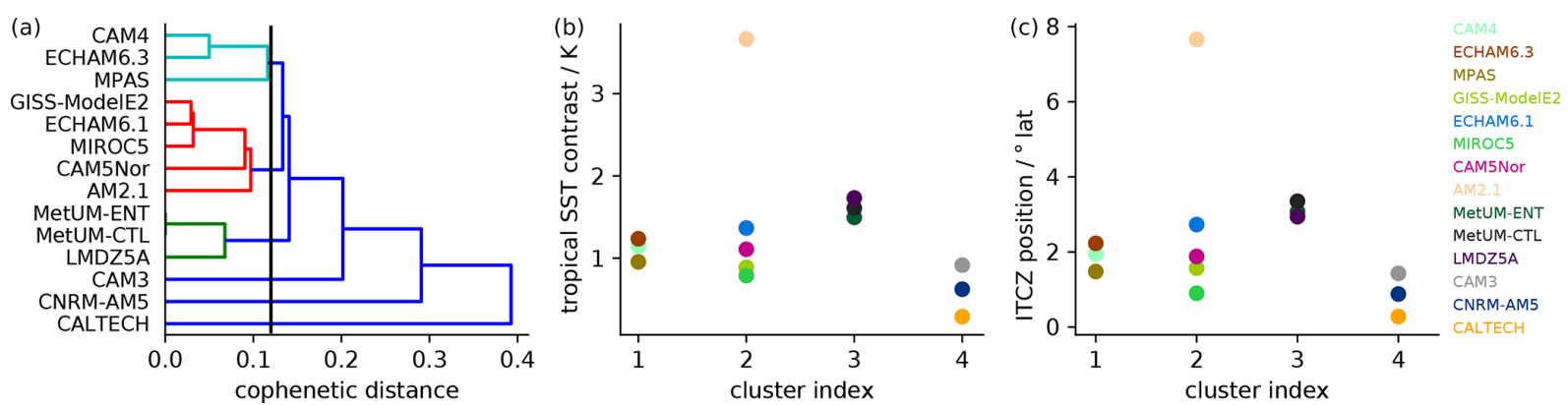

Figure 6. Model clustering based on the AquaControl networks without extratropical-extratropical connections (a) and values of the tropical SST contrast (b) and ITCZ position (c) for all models in the four identified clusters. (a) The dendrogram obtained from the hierarchical clustering of the zonal-mean network measures. The vertical line indicates the level of cophenetic distance at which we split the models into four clusters.
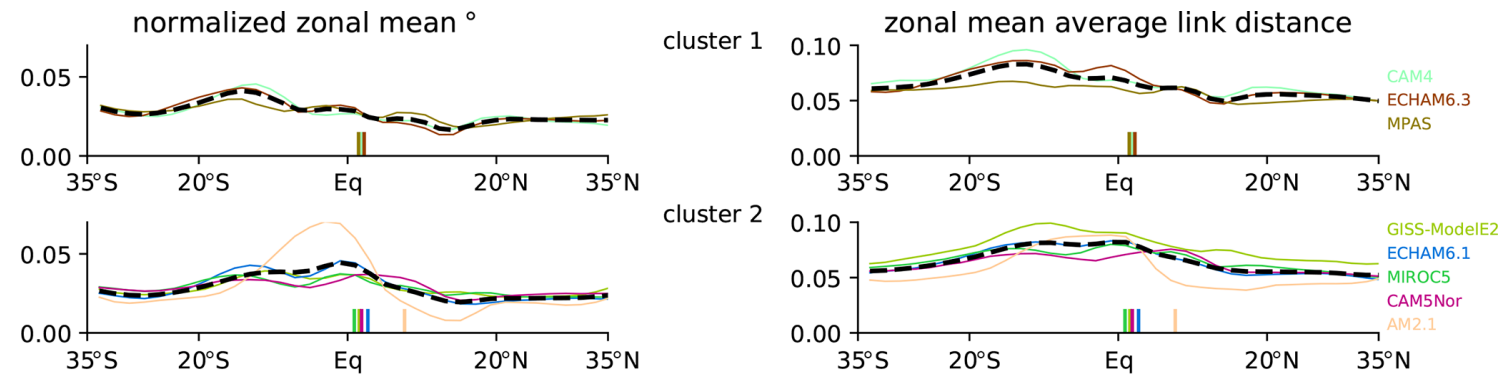

cluster 3
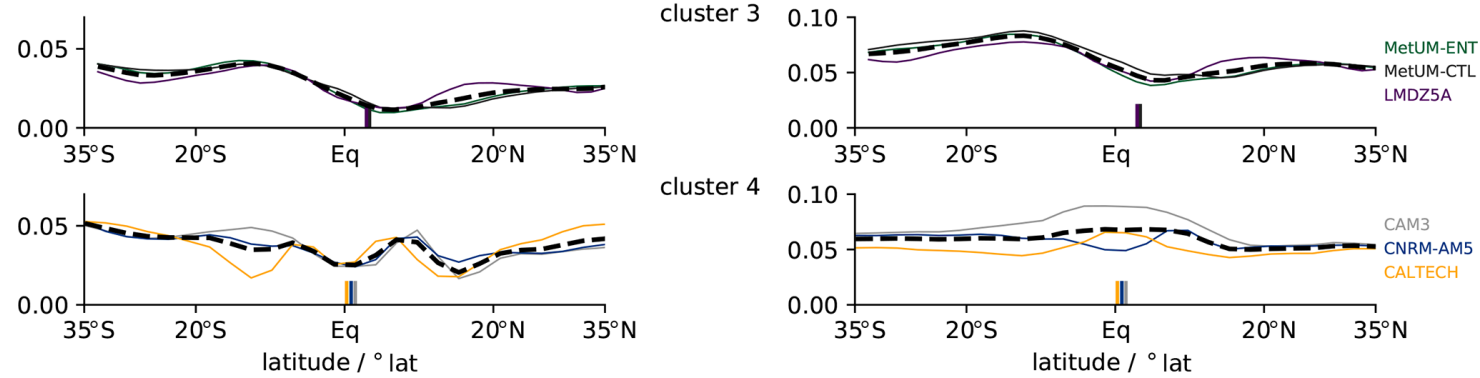

cluster 4

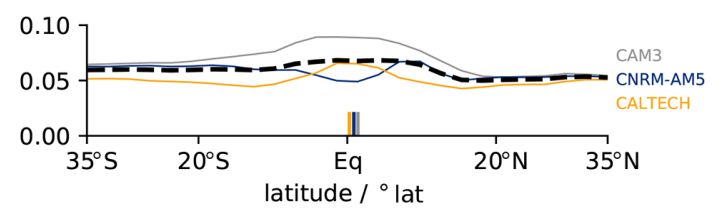

Figure 7. Zonal-mean network characteristics for the AquaControl simulations without extratropical-extratropical connections for each of the four clusters of models. The left panels show the normalized zonal-mean degree, while the right panels display the corresponding average link distances. Vertical lines indicate the respective position of the ITCZ. The cluster means are shown by black dashed lines.

Finally, we also tested the effect of additionally excluding tropical-extratropical connections, thereby retaining only inner-tropical connections. In this case, we did not find coherent clusters with distinct ITCZ positions (not shown). This indicates that tropical-extratropical interactions are important for the ITCZ dynamics (Kang, 2020) but different from time mean frameworks, which typically find a good relation between tropical SST and the ITCZ position (e.g., Donohoe et al., 2013; Biasutti et al., 2018). Furthermore, we have tested our method based on zonal-mean SST fields. Again, this has not led to an interpretable clustering, and the strong decrease in the number of grid points due to the zonal averaging actually turned out to be a challenge to our analysis method. One possible explanation for this is that zonal asymmetries in the tropical circulation, e.g., those related to tropical waves, impact the ITCZ position (Biasutti et al., 2018) and are captured by the network approach, provided that the latter is fed with latitude-longitude data.

\subsection{Aqua4xCO2 - AquaControl simulations: climate response to quadrupling carbon dioxide}

In the previous two subsections, we have demonstrated that functional climate network analysis of monthly SST anomalies can identify model differences in the time average SST contrast and ITCZ position based on the AquaControl simulations. In the following, we determine whether the analysis can also help us to understand model differences in the ITCZ response to global warming triggered by an increase in atmospheric carbon dioxide content in the Aqua $4 \times \mathrm{xCO} 2$ simulations. Across the model ensemble, the ITCZ response 
varies from a slight southward shift by less than $1^{\circ}$ in latitude to a strong northward shift by up to $8^{\circ}$.

We analyze the change in the network measures between the AquaControl and Aqua4xCO2 simulations. We compute the difference between the zonal-mean network characteristics of AquaControl and Aqua4xCO2 for each model, from which we perform the similarity-based hierarchical cluster analysis. The corresponding results are summarized in Figs. 8 and 9. It can be seen that our analysis identifies three clusters. These are presented together with the warminginduced changes in the tropical SST contrasts and ITCZ positions in Fig. 8. Clusters 1 and 2 each consist of two models, and both show a reduced ITCZ response compared to the other models. Cluster 3 contains the majority of models (10 out of 14) and spans the entire ensemble range of ITCZ responses. This wide spread indicates that in contrast to the control climate, the combination of functional network and hierarchical cluster analysis does not pick up model differences in the ITCZ response to global warming. This finding is further illustrated in Fig. 9, which shows the differences in the zonal-mean network properties between the AquaControl and Aqua4xCO2 simulations. While clusters 1 and 2 again both exhibit muted ITCZ responses, they differ substantially in the specific response of their network characteristics. Likewise, for cluster 3 , there is no common spatial pattern in the response of the network measures.

In general, it can be noted that the network analysis appears to fail in capturing inter-model differences in the ITCZ response to warming. In contrast to simulations with realistic present-day boundary conditions, for which the zonal variations in the tropical rainfall response to warming can make it difficult to extract a meaningful zonal-mean response, TRACMIP aquaplanet simulations employ zonally symmetric boundary conditions. The failure of the network analysis must thus have a different origin and indicates that the climate change response of the SST networks is not tightly linked to the ITCZ climate change response. Within the conceptual limitations of the present analysis, the reasons for this behavior remain unclear to us. One possibility could be that unraveling the climate change response would require a different network representation that involves other atmospheric fields in addition to SST, e.g., changes in the vertical profile and gross moist stability of the tropical atmosphere, which Biasutti et al. (2018) suggested to play an important role in TRACMIP. Corresponding follow-up investigations are outlined as a possible subject of future work.

\section{Discussion and conclusions}

The ITCZ is a central element of Earth's climate, yet understanding its dynamics and anticipating its response to climate change remain challenging. In this work, we have proposed a new perspective on the ITCZ by means of complex network theory. We have tested this perspective by analyz- ing a multi-model ensemble of idealized aquaplanet simulations provided by TRACMIP. The main difference between our work and previous considerations of the ITCZ is that our perspective is based on monthly mean anomalies of sea surface temperature (SST), whereas previous work focused on the relation of the time-averaged ITCZ to the time average SST and atmospheric energy transport with a time average that can be seasonal, yearly, or longer.

We have constructed complex network representations based on the correlation pattern of monthly SST anomalies in the control simulation (AquaControl) and a global warming simulation (Aqua4xCO2). We found that the zonal-mean node degree and average link distance of functional climate networks can separate models in terms of their ITCZ position, SST contrast, and Hadley cell strength in the control simulation. This separation also holds when extratropicalextratropical connections are excluded, but it breaks down when further connections are excluded. This shows that the network approach correctly identifies the extratropicaltropical connections as important in setting the tropical climate (Kang, 2020). The network analysis is also consistent with known mechanisms such as a strong correlation between Hadley cell strength and ITCZ position. Overall, the climate network analysis indicates that the time mean ITCZ is connected to spatiotemporal variability of the monthly SST, a finding that is not obvious from previous work. However, we also note that the network analysis was unable to separate model differences in the ITCZ response to global warming.

Because the aquaplanet setup is zonally symmetric in a statistical sense, we have restricted our analysis to zonalmean network properties. This simplification implied the loss of local (single-node) information, which prohibited us from identifying possible local connectivity structures and fully exploiting the complete distribution of links in the network. For example, we have not analyzed if and how individual nodes are connected in the meridional and zonal direction. Future work could look at this aspect in more detail to reveal the role of, e.g., zonally propagating tropical waves associated with large-scale patterns of SST, wind, and atmospheric energy transport. This could also involve other network characteristics like betweenness centrality (Donges et al., 2009) or edge directionality (Wolf et al., 2019), and it would complement traditional empirical orthogonal function (EOF) analysis or more sophisticated pattern recognition approaches such as self-organizing maps (SOMs).

One central goal of this study was to investigate to what extent correlation-based functional climate networks provide insights into the ITCZ dynamics as simulated in state-of-theart global climate models. Some of the results might seem unsurprising, e.g., the fact that an ITCZ closer to the Equator tends to be associated with a more hemispherically symmetric network. In some sense, however, such expected results are reassuring, as they hint at the fact that a network analysis is plausible. Although we have discussed several of 

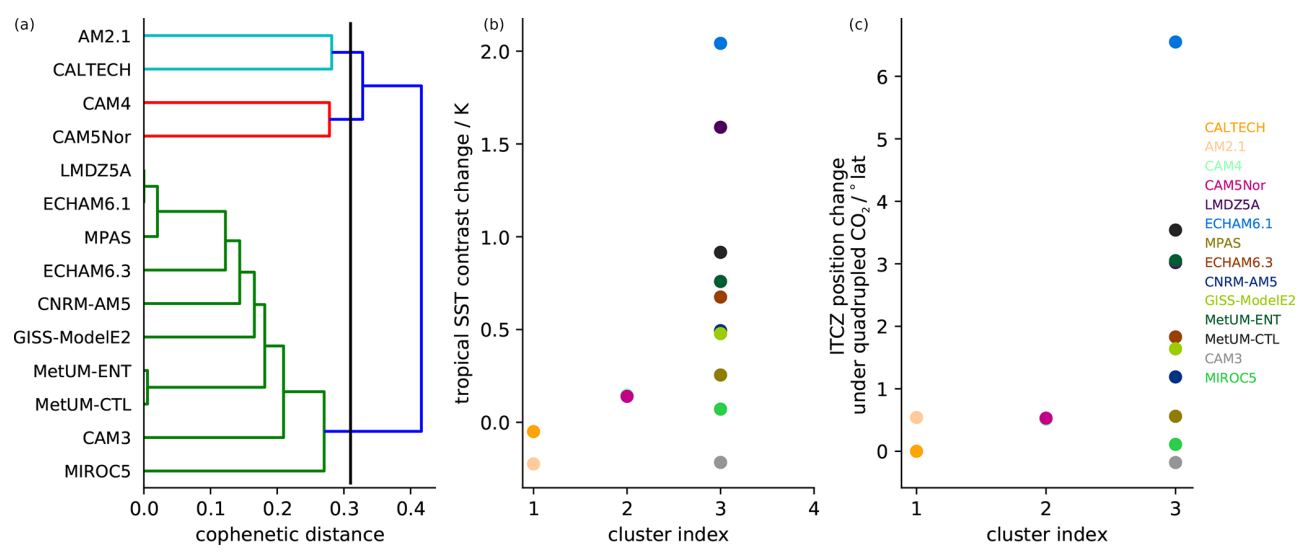

Figure 8. Model clustering based on the difference between the Aqua4xCO2 and AquaControl global networks (a) along with the warminginduced changes in the tropical SST contrast (b) and ITCZ position (c) for the three identified clusters. (a) The dendrogram obtained from the clustering of the difference in the zonal-mean network characteristics. The vertical line indicates the level of cophenetic distance at which we split the models into the three clusters.
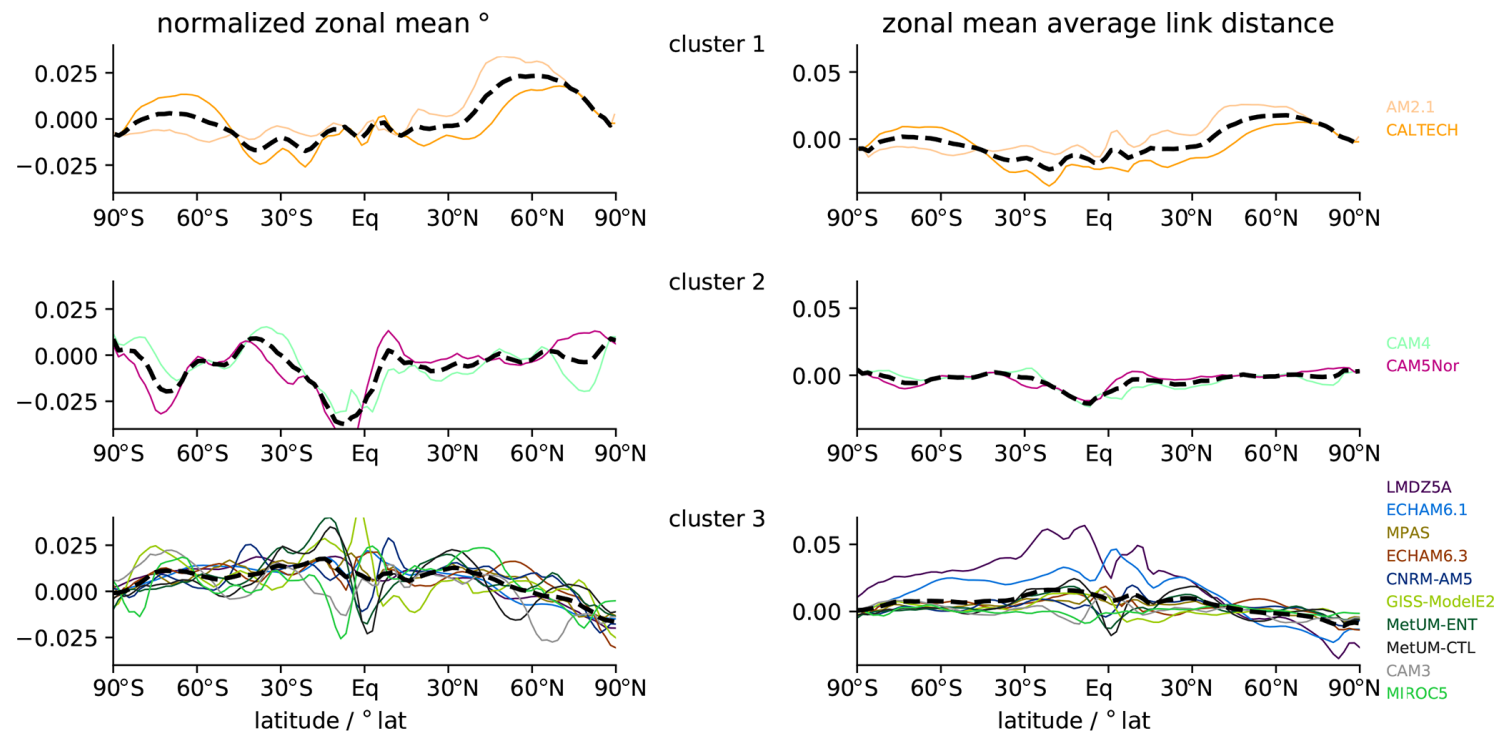

Figure 9. Differences in the zonal-mean network properties of the AquaControl versus Aqua4xCO2 global networks for the three identified clusters. The left panels show the normalized difference between the zonal-mean degrees of the AquaControl and Aqua4xCO2-based networks, while the same is shown in the right panels for the respective average link distances.

the emerging network measures in a climatological context, their implications and specific links to the dynamics of the atmosphere and climate system remain to be further explored. This could include a refinement of the functional network approach to take into account time leads and lags between the ITCZ and regional SST variability. For example, following extratropical perturbations, previous work showed that the extratropical SST response leads those of tropical SST and the ITCZ by several months or even longer, depending on the ocean heat capacity (Woelfle et al., 2015). The network methodology can also be extended in a straightforward manner to include other variables (Donges et al., 2011; Wiedermann et al., 2017a), such as the stratification of the tropical atmosphere and energy fluxes, and to reflect possible leadlag relationships between the ITCZ and the energy budget of the extratropical atmosphere (Adam et al., 2018). In summary, we therefore consider this study a first step towards applications of climate networks and, more broadly, topological data analysis to understand fundamental climate dynamics.

Code and data availability. The Python package pyunicorn (Donges et al., 2015a) used for the functional climate network generation and analysis is freely available at https://github.com/ pik-copan/pyunicorn (last access: 30 March 2021). The TRACMIP data set is available in cmorized format via the Earth System Grid Federation and via the Pangeo Cloud. Instructions on how to use 
TRACMIP via the Pangeo Cloud are given at https://gitlab.phaidra. org/voigta80/tracmip (Voigt and Biasutti, 2021).

Supplement. The supplement related to this article is available online at: https://doi.org/10.5194/esd-12-353-2021-supplement.

Author contributions. All authors contributed to the design of the study. FW implemented the network analysis and the clustering framework. All authors analyzed the results and wrote the paper. All authors read and approved the final paper.

Competing interests. The authors declare that they have no conflict of interest.

Acknowledgements. This work has been supported by the IRTG 1740/TRP 2011/50151-0 (funded by the DFG and FAPESP). Aiko Voigt has received financial support from the German Ministry of Education and Research (BMBF) and FONA (Research for Sustainable Development) under grant agreement 01LK1509A. Aiko Voigt further acknowledges funding from NSF award AGS-1565522. Reik V. Donner acknowledges funding from the German Federal Ministry for Education and Research (BMBF) via the BMBF Young Investigators Group CoSy-CC ${ }^{2}$ (grant no. 01LN1306A), the Belmont Forum/JPI Climate project GOTHAM (grant no. 01LP16MA), and the JPI Climate/JPI Oceans project ROADMAP (grant no. 01LP2002B).

Financial support. This research has been supported by the Deutsche Forschungsgemeinschaft (grant no. IRTG 1740/TRP 2011/50151-0) and the Bundesministerium für Bildung und Forschung (grant nos. 01LN1306A, 01LK1509A, 01LP16MA, and 01LP2002B).

The publication of this article was funded by the Open Access Fund of the Leibniz Association.

Review statement. This paper was edited by Gabriele Messori and reviewed by Anastasios Tsonis and two anonymous referees.

\section{References}

Adam, O., Bischoff, T., and Schneider, T.: Seasonal and Interannual Variations of the Energy Flux Equator and ITCZ. Part I: Zonally Averaged ITCZ Position, J. Climate, 29, 3219-3230, https://doi.org/10.1175/JCLI-D-15-0512.1, 2016.

Adam, O., Schneider, T., and Brient, F.: Regional and seasonal variations of the double-ITCZ bias in CMIP5 models, Clim. Dynam., 51, 101-117, 2018.

Biasutti, M. and Voigt, A.: Seasonal and $\mathrm{CO}_{2}$-Induced Shifts of the ITCZ: Testing Energetic Controls in Idealized Simula- tions with Comprehensive Models, J. Climate, 33, 2853-2870, https://doi.org/10.1175/JCLI-D-19-0602.1, 2020.

Biasutti, M., Voigt, A., Boos, W. R., Braconnot, P., Hargreaves, J. C., Harrison, S. P., Kang, S. M., Mapes, B. E., Scheff, J., Schumacher, C., Sobel, A. H., and Xie, S.-P.: past, present and future monsoons, Nat. Geosci., 11, 392-400, https://doi.org/10.1038/s41561-018-0137-1, 2018.

Boers, N., Bookhagen, B., Marwan, N., Kurths, J., and Marengo, J. A.: Complex networks identify spatial patterns of extreme rainfall events of the South American Monsoon System, Geophys. Res. Lett., 40, 4386-4392, https://doi.org/10.1002/grl.50681, 2013.

Boers, N., Bookhagen, B., Barbosa, H. M. J., Marwan, N., Kurths, J., and Marengo, J. A.: Prediction of extreme floods in the eastern Central Andes based on a complex networks approach, Nat. Commun., 5, 5199, https://doi.org/10.1038/ncomms6199, 2014.

Boers, N., Goswami, B., Rheinwalt, A., Bookhagen, B., Hoskins, B., and Kurths, J.: Complex networks reveal global pattern of extreme-rainfall teleconnections, Nature, 566, 373-377, https://doi.org/10.1038/s41586-018-0872-x, 2019.

Bony, S., Stevens, B., Frierson, D. M. W., Jakob, C., Kageyama, M., Pincus, R., Shepherd, T. G., Sherwood, S. C., Siebesma, A. P., Sobel, A. H., Watanabe, M., and Webb, M. J.: Clouds, circulation and climate sensitivity, Nat. Geeosci., 8, 261-268, https://doi.org/10.1038/ngeo2398, 2015.

Byrne, M. P., Pendergrass, A. G., Rapp, A. D., and Wodzicki, K. R.: Response of the Intertropical Convergence Zone to Climate Change: Location, Width, and Strength, Curr. Clim. Change Rep., 4, 355-370, 2018.

Dijkstra, H. A., Hernández-García, E., Masoller, C., and Barreiro, M.: Networks in Climate, Cambridge University Press, Cambridge, UK, 2019.

Donges, J. F., Zou, Y., Marwan, N., and Kurths, J.: The backbone of the climate network, EPL, 87, 48007, https://doi.org/10.1209/0295-5075/87/48007, 2009.

Donges, J. F., Schultz, H. C., Marwan, N., Zou, Y., and Kurths, J.: Investigating the topology of interacting networks: Theory and application to coupled climate subnetworks, Eur. Phys. J. B, 84, 635-651, https://doi.org/10.1140/epjb/e2011-10795-8, 2011.

Donges, J. F., Heitzig, J., Beronov, B., Wiedermann, M., Runge, J., Feng, Q. Y., Stolbova, V., Donner, R. V., Marwan, N., Dijkstra, H. A., and Kurths, J.: Unified functional network and nonlinear time series analysis for complex systems science: The pyunicorn package, Chaos, 25, 113101, https://doi.org/10.1063/1.4934554, 2015a.

Donges, J. F., Petrova, I., Loew, A., Marwan, N., and Kurths, J.: How complex climate networks complement eigen techniques for the statistical analysis of climatological data, Clim. Dynam., 45, 2407-2424, https://doi.org/10.1007/s00382-015-2479$3,2015 b$.

Donner, R. V., Wiedermann, M., and Donges, J. F.: Complex Network Techniques for Climatological Data Analysis, in: Nonlinear and Stochastic Climate Dynamics, edited by: Franzke, C. and O'Kane, T., Cambridge University Press, Cambridge, 159-183, 2017.

Donohoe, A. and Voigt, A.: Why Future Shifts in Tropical Precipitation Will Likely Be Small, in: Climate Extremes: Patterns and Mechanisms, edited by: Wang, S.-Y. S., Yoon, J.-H., Funk, 
C., and Gillies, R., Academic Press, Cambridge, Massachusetts, USA, 115-137, 2017.

Donohoe, A., Marshall, J., Ferreira, D., and McGee, D.: The Relationship between ITCZ Location and Cross-Equatorial Atmospheric Heat Transport: From the Seasonal Cycle to the Last Glacial Maximum, J. Climate, 26, 3597-3618, https://doi.org/10.1175/JCLI-D-12-00467.1, 2013.

Emanuel, K. A., Neelin, J. D., and Bretherton, C. S.: On large-scale circulations in convecting atmospheres, Q. J. Roy. Meteor. Soc., 120, 1111-1143, 1994.

Fan, J., Meng, J., Ludescher, J., Li, Z., Surovyatkina, E., Chen, X., Kurths, J., and Schellnhuber, H. J.: Network-based Approach and Climate Change Benefits for Forecasting the Amount of Indian Monsoon Rainfall, arXiv [preprint], arXiv:2004.06628, 2020.

Feng, Q. Y., Vasile, R., Segond, M., Gozolchiani, A., Wang, Y., Abel, M., Havlin, S., Bunde, A., and Dijkstra, H. A.: ClimateLearn: A machine-learning approach for climate prediction using network measures, Geosci. Model Dev. Discuss. [preprint], https://doi.org/10.5194/gmd-2015-273, 2016.

Frierson, D., Hwang, Y., and Fuckar, N.: Contribution of ocean overturning circulation to tropical rainfall peak in the Northern Hemisphere, Nature Geosci., 6, 940-944, 2013.

Gozolchiani, A., Yamasaki, K., Gazit, O., and Havlin, S.: Pattern of climate network blinking links follows El Niño events, EPL (Europhysics Letters), 83, 28005, https://doi.org/10.1209/02955075/83/28005, 2008.

Gozolchiani, A., Havlin, S., and Yamasaki, K.: Emergence of El Niño as an Autonomous Component in the Climate Network, Phys. Rev. Lett., 107, 148501, https://doi.org/10.1103/PhysRevLett.107.148501, 2011.

Harrison, S. P., Bartlein, P. J., Izumi, K., Li, G., Annan, J., Hargreaves, J., Braconnot, P., and Kageyama, M.: Evaluation of CMIP5 palaeo-simulations to improve climate projections, Nat. Clim. Change, 5, 735-743, https://doi.org/10.1038/NCLIMATE2649, 2015.

Heitzig, J., Donges, J. F., Zou, Y., Marwan, N., and Kurths, J.: Nodeweighted measures for complex networks with spatially embedded, sampled, or differently sized nodes, Eur. Phys. J. B, 85, 38, https://doi.org/10.1140/epjb/e2011-20678-7, 2012.

Hwang, Y., Frierson, D., and Kang, S. M.: Anthropogenic sulfate aerosol and the southward shift of tropical precipitation in the late 20th century, Geophys. Res. Lett., 40, 2845-2850, 2013.

Kang, S. M.: Extratropical Influence on the Tropical Rainfall Distribution, Curr. Clim. Chang. Rep., 6, 24-36, 2020.

Kang, S. M., Frierson, D. M. W., and Held, I. M.: The Tropical Response to Extratropical Thermal Forcing in an Idealized GCM: The Importance of Radiative Feedbacks and Convective Parameterization, J. Atmos. Sci., 66, 2812-2827, https://doi.org/10.1175/2009JAS2924.1, 2009.

Kang, S. M., Shin, Y., and Xie, S.-P.: Extratropical forcing and tropical rainfall distribution: energetics framework and ocean Ekman advection, NPJ Clim. Atmos. Sci., 1, 20172, https://doi.org/10.1038/s41612-017-0004-6, 2018.

Lindzen, R. S. and Nigam, S.: On the Role of Sea Surface Temperature Gradients in Forcing Low-Level Winds and Convergence in the Tropics, J. Atmos. Sci., 44, 2418-2436, 1987.

Ludescher, J., Gozolchiani, A., Bogachev, M. I., Bunde, A., Havlin, S., and Schellnhuber, H. J.: Improved El Niño forecasting by cooperativity detection, P. Natl. Acad. Sci. USA, 110, 1174211745, https://doi.org/10.1073/pnas.1309353110, 2013.

Ludescher, J., Gozolchiani, A., Bogachev, M. I., Bunde, A., Havlin, S., and Schellnhuber, H. J.: Very early warning of next El Niño, P. Natl. Acad. Sci. USA, 111, 2064-2066, https://doi.org/10.1073/pnas.1323058111, 2014.

Malik, N., Bookhagen, B., Marwan, N., and Kurths, J.: Analysis of spatial and temporal extreme monsoonal rainfall over South Asia using complex networks, Clim. Dynam., 39, 971987, https://doi.org/10.1007/s00382-011-1156-4, 2012.

Meng, J., Fan, J., Ludescher, J., Agarwal, A., Chen, X., Bunde, A., Kurths, J., and Schellnhuber, H. J.: Complexity-based approach for El Niño magnitude forecasting before the spring predictability barrier, P. Natl. Acad. Sci. USA, 117, 177-183, https://doi.org/10.1073/pnas.1917007117, 2020.

Moebis, B. and Stevens, B.: Factors controlling the position of the Intertropical Convergence Zone on an aquaplanet, J. Adv. Model. Earth Syst., 4, https://doi.org/10.1029/2012MS000199, 2012.

Radebach, A., Donner, R. V., Runge, J., Donges, J. F., and Kurths, J.: Disentangling different types of El Niño episodes by evolving climate network analysis, Phys. Rev. E, 88, 052807, https://doi.org/10.1103/PhysRevE.88.052807, 2013.

Russotto, R. D. and Biasutti, M.: Polar Amplification as an Inherent Response of a Circulating Atmosphere: Results From the TRACMIP Aquaplanets, Geophys. Res. Lett., 47, e2019GL086771, https://doi.org/10.1029/2019GL086771, 2020.

Schneider, T., Bischoff, T., and Haug, G. H.: Migrations and dynamics of the intertropical convergence zone, Nature, 513, 4553, https://doi.org/10.1038/nature13636, 2014.

Stolbova, V., Martin, P., Bookhagen, B., Marwan, N., and Kurths, J.: Topology and seasonal evolution of the network of extreme precipitation over the Indian subcontinent and Sri Lanka, Nonlin. Processes Geophys., 21, 901-917, https://doi.org/10.5194/npg21-901-2014, 2014.

Stolbova, V., Surovyatkina, E., Bookhagen, B., and Kurths, J.: Tipping elements of the Indian monsoon: Prediction of onset and withdrawal, Geophys. Res. Lett., 43, 3982-3990, https://doi.org/10.1002/2016GL068392, 2016.

Tsonis, A. A. and Roebber, P. J.: The architecture of the climate network, Phys. A, 333, 497-504, 2004.

Tsonis, A. A. and Swanson, K. L.: Topology and predictability of El Niño and la Niña Networks, Phys. Rev. Lett., 100, 228502, https://doi.org/10.1103/PhysRevLett.100.228502, 2008.

Tsonis, A. A., Swanson, K. L., and Roebber, P. J.: What do networks have to do with climate?, B. Am. Meteorol. Soc., 87, 585-595, 2006.

van der Mheen, M., Dijkstra, H. A., Gozolchiani, A., den Toom, M., Feng, Q., Kurths, J., and Hernandez-Garcia, E.: Interaction network based early warning indicators for the Atlantic MOC collapse, Geophys. Res. Lett., 40, 2714-2719, https://doi.org/10.1002/grl.50515, 2013.

Voigt, A. and Biasutti, M.: TRACMIP data set, available at: https:// gitlab.phaidra.org/voigta80/tracmip, last access: 30 March 2021.

Voigt, A., Bony, S., Dufresne, J.-L., and Stevens, B.: The radiative impact of clouds on the shift of the Intertropical Convergence Zone, Geophys. Res. Lett., 41, 4308-4315, https://doi.org/10.1002/2014GL060354, 2014.

Voigt, A., Biasutti, M., Scheff, J., Bader, J., Bordoni, S., Codron, F., Dixon, R. D., Jonas, J., Kang, S. M., Klingaman, N. P., Leung, 
R., Lu, J., Mapes, B., Maroon, E. A., McDermid, S., Park, J., Roehrig, R., Rose, B. E. J., Russell, G. L., Seo, J., Toniazzo, T., Wei, H., Yoshimori, M., and Vargas Zeppetello, L. R.: The tropical rain belts with an annual cycle and a continent model intercomparison project: TRACMIP, J. Adv. Model. Earth Sy., 8, 1868-1891, 2016.

Voigt, A., Pincus, R., Stevens, B., Bony, S., Boucher, O., Bellouin, N., Lewinschal, A., Medeiros, B., Wang, Z., and Zhang, H.: Fast and slow shifts of the zonal-mean intertropical convergence zone in response to an idealized anthropogenic aerosol, J. Adv. Model. Earth Sy., 9, 870-892, 2017.

Wallace, J. and Hobbs, P.: Atmospheric Science, Academic Press, Cambridge, Massachusetts, USA, 2006.

Wiedermann, M., Radebach, A., Donges, J. F., Kurths, J., and Donner, R. V.: A climate network-based index to discriminate different types of El Niño and La Niña, Geophys. Res. Lett., 43, 7176-7185, https://doi.org/10.1002/2016GL069119, 2016.

Wiedermann, M., Donges, J. F., Handorf, D., Kurths, J., and Donner, R. V.: Hierarchical structures in Northern Hemispheric extratropical winter ocean-atmosphere interactions, Int. J. Climatol., 37, 3821-3836, 2017a.
Wiedermann, M., Donges, J. F., Kurths, J., and Donner, R. V.: Mapping and discrimination of networks in the complexity-entropy plane, Phys. Rev. E, 96, 042304, https://doi.org/10.1103/PhysRevE.96.042304, 2017b.

Woelfle, M. D., Bretherton, C. S., and Frierson, D. M.: Time scales of response to antisymmetric surface fluxes in an aquaplanet GCM, Geophys. Res. Lett., 42, 2555-2562, 2015.

Wolf, F., Kirsch, C., and Donner, R. V.: Edge directionality properties in complex spherical networks, Phys. Rev. E, 99, 012301, https://doi.org/10.1103/PhysRevE.99.012301, 2019.

Yamasaki, K., Gozolchiani, A., and Havlin, S.: Climate networks around the globe are significantly affected by El Niño, Phys. Rev. Lett., 100, 228501, https://doi.org/10.1103/PhysRevLett.100.228501, 2008.

Zhou, D., Gozolchiani, A., Ashkenazy, Y., and Havlin, S.: Teleconnection Paths via Climate Network Direct Link Detection, Phys. Rev. Lett., 115, 268501, https://doi.org/10.1103/PhysRevLett.115.268501, 2015. 